\title{
Vernetzte Spitzenforschung entwickelt neue Therapien für Atemwegserkrankungen
}

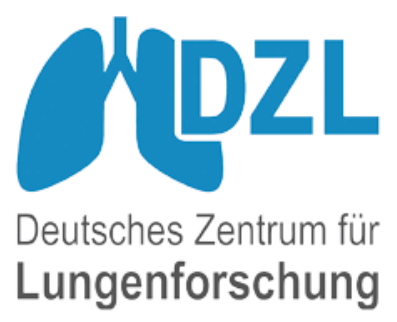

\author{
Vor fünf Jahren hat sich eine Reihe exzellenter Forschungseinrichtungen zum Deutschen \\ Zentrum für Lungenforschung vernetzt. Beim DGP-Kongress 2016 zog man eine positive Bilanz.
}

\begin{abstract}
„Wir sind Top in der Grundlagenforschung mit Publikationen in Science, Nature oder Cell, bringen aber unsere Ergebnisse zu langsam an den Patienten" - dieser Satz von Prof. Tobias Welte beschreibt ein Forschungsdilemma der Vergangenheit, welches das Bundesministerium für Bildung und Forschung im Jahr 2011 veranlasst hat, die translationale Forschung in sechs Kerngebieten der Medizin zu fördern, darunter die Pneumologie.

Entstanden ist das Deutsche Zentrum für Lungenforschung* (DZL), ein Verbund aus fünf Standorten mit 23 Partnerinstitutionen. Der klare Auftrag lautete, dass sich die besten Forschungsinstitutionen Deutschlands vernetzen und gemeinsam translationale Forschung vorantreiben sollen. Es galt zunächst zügig Strukturen zu schaffen, um neue Substanzen und Devices für die Patienten entwickeln zu können.

Beim DGP-Kongress zog Welte, Direktor der Klinik für Pneumologie der Med. Hochschule Hannover und DZL-Standortleiter in Hannover, eine positive Bilanz der bisherigen Arbeit: „Es ist gelungen, wesentliche Strukturen für eine vernetzte translationale Forschung aufzubauen. Wir haben ein gemeinsames Datenmanagement etabliert, ein gemeinsames Umgehen mit Biomaterialdatenbanken, mit Bilddatenbanken, und wir haben ein absolut sicheres Datenschutzkonzept entwickelt. Drei Viertel aller Projekte sind standortübergreifende Gemeinschaftsprojekte.“
\end{abstract}

\section{Makrophagen-Wachstumsfaktoren bei ARDS}

$\mathrm{Zu}$ fast allen großen pneumologischen Krankheitsbildern wurden große Kohorten und Register geschaffen, mit deren Hilfe sowohl die aktuelle Versorgung evaluiert als auch neue Medikamente getestet werden können. Ein wesentlicher Gesichtspunkt sei die Zusammenarbeit mit Forschungszentren anderer
Disziplinen, so Welte. Es gibt gemeinsame Projekte mit dem Deutschen Zentrum für Infektionsforschung, etwa bei den Pneumonien, dem Deutschen Zentrum für Herz-Kreislaufforschung, etwa bei der Pulmonalen Hypertonie, und dem Deutschen Zentrum für Krebsforschung beim Lungenkrebs. Auch mit der Fachgesellschaft DGP ist man sehr eng vernetzt.

Welte nannte zwei Beispielprojekte, die in absehbarer Zukunft einen Nutzen für Patienten bringen könnten. In der Intensivmedizin forscht man an Makrophagen-Wachstumsfaktoren, die beim „acute respiratory distress syndrome“ (ARDS) direkt in die Lungen installiert werden. Eine erste Phase IIa-Studie beim Menschen ist bereits gestartet.

\section{Künstliche Lunge für die Langzeitanwendung}

Das zweite Projekt betrifft die Entwicklung einer künstlichen Lunge. Welte: „Wir haben heute Möglichkeiten geschaffen, eine extrakorporale Sauerstoff-Anreicherung und KohlendioxidEntfernung für längere Zeit zu ermöglichen. Nun arbeiten wir daran, die Miniaturisierung weiter zu verbessern, die Blutseite der Membran mit menschlichen Epithelzellen zu beschichten und die Anschlüsse an das Gefäßsystem zu bessern. Persönlich glaube ich daran, dass wir in den nächsten zehn Jahren einen ersten chronischen Lungenersatz hinbekommen werden." (DE)

\footnotetext{
*Dem DZL gehören 5 Standortverbunde mit 23 Partnerinstitutionen an:

1. Comprehensive Pneumology Center in München,

2. Translational Lung Research Center in Heidelberg,

3. Biomedical Research in Endstage and Obstructive Lunge Disease in Hannover,

4. Universities of Giessen and Marburg Lung Center (mit Bad Nauheim),

5. Airway Research Center North in Borstel, Lübeck, Kiel und Großhansdorf.
}

\section{Kontrolliert beatmen - aber richtig}

Die kontrollierte Beatmung gehört zu den schwierigsten Therapien in der Notfallmedizin. Zu den zehn wichtigsten Standardparametern, die eingestellt werden müssen, gehört der positive endexspiratorische Druck (PEEP). Doch wie hoch soll er sein?

Der PEEP kann Gasaustausch und Ventilation über den Einfluss auf verschiedene Parameter verbessern, etwa durch die Abnahme des intrapulmonalen Rechts-Links-Shunts und Abnahme der Atemarbeit, die Verbesserung des Ventilations-Perfusions-Verhältnisses sowie die Zunahme der funktionellen Residualkapazität durch Prävention von De-Rekrutierung. Unklar ist allerdings noch immer, ob PEEP zu einer Stabilisierung der Atemwege führt, mit Abnahme der exspiratorischen Flusslimitationen und auch einer Reduktion der dynamischen Überblähung.

Und: PEEP ist nicht ohne Nebenwirkungen. So kann es bei inadäquat hohem PEEP u. a. zu einem Überdehnungsschaden gut ventilierter Alveolen kommen, zu einer Totraumventilation durch Kapillarkompression oder auch zu einem erhöhten intrathorakalen Druck. Lebervenendruck und Pfortaderdruck können ansteigen. „Die PEEP-Wahl ist immer noch ein kritischer Punkt und ein klinisches Problem”, erklärt Dr. Simone Rosseau, Leiterin des Charité Zentrum für ambulante Beatmungsmedizin und Sauerstofftherapie (CABS). Sie plädierte für einen „ausreichend hohen PEEP”.

Problem: De-Rekrutierung

Generell stehen bei der kontrollierten Beatmung zwei Verfahren zur Verfügung: die druckkontrollierte und volumenkontrollierte CMV („Continuous mandatory ventilation“). Am häufigsten wird die druckkontrollierte CMV eingesetzt. Doch beide Methoden haben Vor- und Nachteile. Rosseau sieht als Vorteil der volumenkontrollierten CMV die genaue Kontrolle von Atemzugvolumen und Atemminutenvolumen, vor allem aber ein geringeres Risiko der De-Rerekrutierung, denn: „De-Rekrutierung kann zu einem Beatmungs-induzierten Lungenschaden führen."

Beate Fessler 